\title{
CHEST What's hot that the CLINIC others got
}

\author{
Janice Higginson
}

\section{LINIFANIB IN STAGE IIIB OR IV NON-SMALL CELL LUNG CANCER}

Platinum-based chemotherapy is standard treatment for non-small cell lung cancer (NSCLC), but in the expanding world of tyrosine kinase inhibitors established regimes are being questioned. Ramalingham et al (J Clin Oncol 2015;33:433-41) have published a Phase II randomised, doubleblind, placebo-controlled multicentre trial studying the effect of adding linifanib to carboplatin and paclitaxel. Patients with stage IIIB or IV NSCLC and performance status 1 or below were carefully selected and then randomised to three arms: carboplatin and paclitaxel plus placebo, $7.5 \mathrm{mg}$ linifanib or $12.5 \mathrm{mg}$ linifanib. Progression-free survival (PFS) was significantly improved in the linifanib $7.5 \mathrm{mg}$ arm compared with the $12.5 \mathrm{mg}$ and placebo arms (252, 221 and 174 days, respectively). Overall survival was not different between the arms, although the study was underpowered for this outcome. There was a significant improvement of PFS in the linifanib arms in a subgroup of patients with a positive CEA/CYFRA 21-1 biomarker signature. Adverse events were higher in the linifanib arms, including thrombocytopenia and two deaths, as well as dose-related diarrhoea, anaemia, hypertension, dysphonia, decreased weight and palmar-plantar erythrodysesthesia. The authors conclude that linifanib does show efficacy when added to carboplatin and paclitaxel and that further study into the CEA/CYFRA 21-1-positive subgroup is warranted.

\section{ROFLUMILAST TO PREVENT EXACERBATIONS IN SEVERE COPD}

Roflumilast is an oral phosphodiesterase-4 inhibitor with anti-inflammatory properties. The REACT trial (Lancet 2015. Published Online First: 13 February, http:/dx.doi.org/ $10.1016 / \mathrm{S} 0140-6736(14) 62410-7)$ is a 1-year double-blind, placebo-controlled multicentre trial targeting patients

Correspondence to Dr Janice Higginson, ST4 Respiratory and General Medicine, James Cook University Hospital, North Yorkshire, UK; janice.higginson@nhs.net with severe COPD and frequent exacerbations. Frequency of severe exacerbations and hospitalisations were reduced with Roflumilast compared with placebo in the negative binomial regression (RR 0.757 (95\% CI 0.601 to 0.952$), p=0.0175)$ and intention-to-treat analysis (RR 0.761 (95\% CI 0.604 to 0.960$), p=0.0209$ ), respectively. A significant increase in $\mathrm{FEV}_{1}$ was seen in the Roflumilast group at 12 weeks and sustained to 52 weeks with a difference at 52 weeks of $56 \mathrm{~mL}(38-73) ; \mathrm{p}<0.0001$. Twenty-six per cent of the Roflumilast group reported adverse events and 11\% withdrew, and $30 \%$ of the placebo group reported adverse events and 5\% withdrew. The authors recognise that the study of exacerbation frequency is challenging, but important; in an exacerbating phenotype, Roflumilast has the potential to improve outcomes.

\section{SELECTIVE LYMPH NODE RESECTION AT LOBECTOMY FOR NSCLC}

Riquet et al (Eur J Cardiothorac Surg $2015 ; 47: 543-9)$ aim to answer the question of whether selective lymph node clearance during surgery for NSCLC can be practised safely. One thousand seven hundred and seventy-nine lobectomy cases from two French centres between 1980 and 2009 were reclassified with the 2010 international staging system for NSCLC and reviewed. Tumour locations were split into Group 1: right upper, Group 2: middle, Group 3: right lower lobe (RLL), Group 4: left upper division segment, Group 5: lingua and Group 6: left lower lobe. Tumour location was mapped to positive node station and although the closest lymph node was involved in the majority of cases, this was not exclusive; for example, in RLL tumours, $10 \%$ of single-station $\mathrm{N} 2$ was found in the superior mediastinum. Survival was significantly affected by nodal stage with 5 -year survival of N2 disease of $30.9 \%$ compared with $65 \%$ of N0. Multi-station N2 disease had worse 5-year survival compared with N0N1 or N1N2 skip metastasis. There was no significant survival difference between left side lobectomy and right side lobectomy except for significantly improved RUL survival compared with LUL survival in N1 disease. The authors conclude that selective lymph node resection would not reliably treat lymph node disease and would detrimentally affect survival. The current practice of ipsilateral lymph node clearance at lobectomy for NSCLC should continue.

\section{INTENSIVE CARE AND HOSPITAL MORTALITY IN OBSTRUCTIVE SLEEP APNOEA PATIENTS}

Patients with obstructive sleep apnoea (OSA) have higher incidence of comorbid conditions such as diabetes, obesity and ischaemic heart disease; they are also more likely to develop type 2 respiratory failure due to obesity hypoventilation. Theoretically therefore, patients with OSA should be more likely to be admitted to integrated critical care (ICU) and have a higher mortality. Bolona et al (J Crit Care $2015 ; 30: 178-80)$ tested this hypothesis by reviewing 15077 cases admitted to medical, surgical or mixed ICU in the Mayo Clinic from 2003 to 2005 . The total prevalence of OSA was $7.8 \%$, patients with OSA were younger $(59.1 \pm 14.0)$ compared with the group without OSA $(62.3 \pm 18.0)$ and were more likely to be men $(58.9 \%$ vs 53.7\%). Patients with OSA had a lower APACHE III score $(45.3 \pm 24.1$ vs 54.9 $\pm 27.7)$, lower acute physiology score (35.3 \pm 21.1 vs $41.8 \pm 24.7)$ and lower predicted mortality $\quad(10.3 \% \pm 16.4 \% \quad$ vs $16.3 \%$ $\pm 21.7 \%$ ), all with $\mathrm{p}$ values below 0.001 . ICU mortality $(2.4 \%$ vs $6.2 \%)$ and hospital mortality $(3.9 \%$ vs $11.4 \%)$ were both significantly lower for patients with a diagnosis of OSA. After adjusting APACHE III scores, the OR for mortality in the OSA group was 0.408 (95\% CI 0.298 to 0.557 ) compared with the group without OSA. These results suggest that OSA is independently associated with reduced hospital mortality. The authors postulate that these unexpected findings could be due to the protective effects of obesity in critical illness or a lower threshold to admit patients with OSA to ICU.

\section{Competing interests None.}

Provenance and peer review Commissioned; internally peer reviewed.

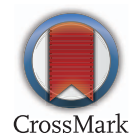

To cite Higginson J. Thorax 2015;70:514.

Thorax 2015;70:514.

doi:10.1136/thoraxjnl-2015-207091 